\title{
Improving the quality of H.264/AVC by using a new Rate-Quantization model
}

\author{
Hrarti M. ${ }^{12}$, Saadane H. ${ }^{13}$, Larabi M-C. ${ }^{1}$, Tamtaoui A.4 and Aboutajdine D. ${ }^{2}$ \\ ${ }^{1}$ XLim Institute, SIC Laboratory, University of Poitiers, Marie et Pierre Currie Bvd, B. P. \\ 30179-86962 Futuroscope, Poitiers, France. \\ ${ }^{2}$ GSCM-LRIT Laboratory, Faculty of Sciences, University MohamedV, Ibn Battouta Avenue \\ B.P. 1014, Rabat, Morocco. \\ ${ }^{3}$ Polytechnic School of Nantes University, Nantes, France. \\ 4LTI Laboratory, National Institute of Post and Telecommunications, Rabat, Morocco.
}

\begin{abstract}
Rate control plays a key role in video coding standards. Its goal is to achieve a good quality at a given target bit-rate. In H.264/AVC, rate control algorithm for both Intra and Inter-frames suffers from some defects. In the Intra-frame rate control, the initial quantization parameter (QP) is mainly adjusted according to a global target bit-rate and length of GOP. This determination is inappropriate and generates errors in the whole of video sequence. For Inter coding unit (Frame or Macroblock), the use of MAD (Mean Average Differences) as a complexity measure, remains inefficient, resulting in improper QP values because the MAD handles locally images characteristics. QP miscalculations may also result from the linear prediction model which assumes similar complexity from coding unit to another. To overcome these defects, we propose in this paper, a new Rate-Quantization (R-Q) model resulting from extensive experiments. This latter is divided into two models. The first one is an Intra R-Q model used to determine an optimal initial quantization parameter for Intraframes. The second one is an Inter R-Q model that aims at determining the QP of Inter coding unit according to the statistics of the previous coded ones. It does not use any complexity measure and substitutes both linear and quadratic models used in H.264/AVC rate controller. Objective and subjective simulations have been carried out using JM15.0 reference software. Compared to this latter, the global R-Q model (Intra and Inter models combined) improves the coding efficiency in terms of PSNR, objectively (up to $+2.01 \mathrm{~dB}$ ), subjectively (by psychophysical experiments) and in terms of computational complexity.
\end{abstract}

Keywords: H.264/AVC Standard, Complexity measure, Rate-Quantization model, Inter/ Intra Frame, Rate control algorithm.

\section{INTRODUCTION}

H.264/AVC (Advanced Video Coding) is the newest international video coding standard that provides approximately a $50 \%$ bit-rate savings for equivalent perceptual quality relative to the performance of prior standards. This is because it has many outstanding features, such as various Intra/Inter prediction modes, 4x 4 integer transform, rate-distortion optimization, and improved entropy coding ${ }^{13}$. These new techniques do not consider the issue of maintaining a consistant quality and a Constant Bit-Rate (CBR) through the network channel. Hence, it is necessary to adjust dynamically the quantization parameter (QP) by implementing a rate control algorithm in the video encoder.

Figure 1 illustrates the two main steps in H.264/AVC rate control process: Bit allocation scheme (at frame or basic unit layers) that uses MPEG2 TM5 ${ }^{4}$ as a benchmark, and the bit allocation achievement, i.e., quantization parameter determination using quadratic Rate-Quantization (R-Q) model.

It should be noted that in Figure 1, we do not consider B-frame due to the fact that this latter takes up only a little part in total encoded bit-stream, which can be ignored in low bit-rate. Therefore, we only consider I- and

Further author information: (Send correspondence to Hrarti M.)

Hrarti M.: E-mail: hrarti@sic.sp2mi.univ-poitiers.fr

Image Quality and System Performance VIII, edited by Susan P. Farnand, Frans Gaykema, Proc. of SPIE-IS\&T Electronic Imaging, SPIE Vol. 7867, 78670J · C 2011 SPIE-IS\&T · CCC code: 0277-786X/11/\$18 · doi: 10.1117/12.872426 


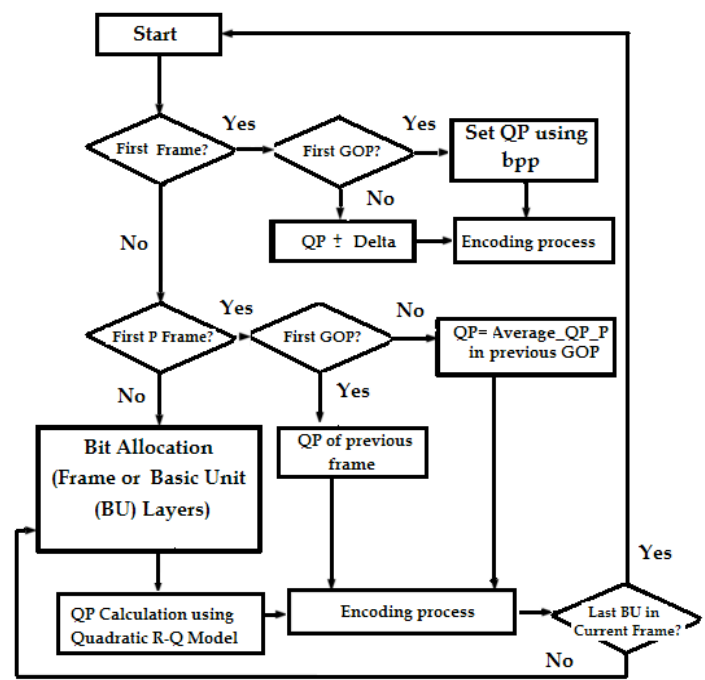

Figure 1. H.264/AVC Rate control schema for both I-frame and P-Basic Unit

P-frames in this paper. Rate control algorithms in previous video coding standards such as MPEG2 TM5 ${ }^{4}$, H.263 $\mathrm{TMN}^{6}$ and MPEG4 VMN8 ${ }^{10}$ are simpler than that of H.264/AVC, because this latter applies the Lagrangian method (or RDO: Rate-Distortion Optimisation) which requires the QP to be known before motion estimation and mode decision, thus leading to a chicken and egg dilemma because until the end of motion estimation and mode decision, the rate control algorithm cannot access the statistics such as MAD (Mean Average Difference), which are essential to calculate QP. It can be also seen from Figure 1, that the rate control process is different for both Intra-frame (I-frame) and Inter coding unit.

In the Intra-rate control, an initial QP is determined for the I-frame and the first P-frame in a given GOP. This selection is given in the following flowchart:

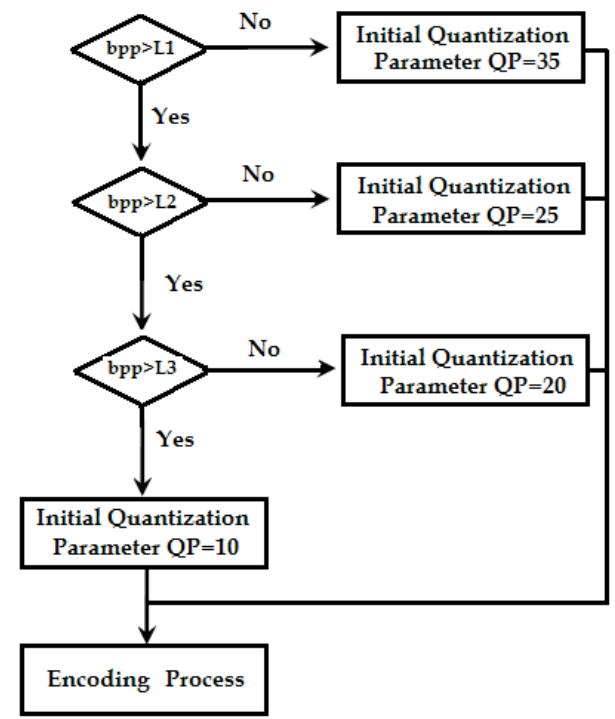

Figure 2. Flowchart of the Initial QP selection process in H.264/AVC Intra-rate control. 
where

$$
b p p=\frac{\text { GlobalBitrate }}{F r \times \text { image_height } \times \text { image_width }}
$$

bpp is the number of bits per pixel, GlobalBitrate is the global target bit-rate for the whole sequence and $\mathrm{Fr}$ is the frame rate. $L_{1}, L_{2}$, and $L_{3}$ are defined in ${ }^{9}$.

As mentioned above, this initial QP is adjusted mainly according to global target bit-rate (GlobalBitrate) and length of GOP. This approximative choice generates errors that are propagated in the whole of video sequence by mean of motion estimation and motion compensation frameworks used in H.264/AVC. To overcome this problem, several Intra-rate control studies have been conducted ${ }^{1} 812$. The proposed solutions however, present two difficulties, to be applied directly in H.264/AVC. Firstly, they need information after actually encoding the current frame to determine the appropriate QP which does not comply with H.264/AVC RDO procedure. Secondly, they need a lot of additional computations for a complexity measure including I-frame feature ${ }^{11} 18$.

In the H.264/AVC Inter-rate control algorithm, the chicken and egg dilemma is always present. To get around this problem, Li et $\mathrm{al}^{17}$ propose a linear model. This latter assumes that the complexity calculated by MAD (Mean Absolute Difference), varies gradually between the current and the previous coding unit (Frame or Macroblock).

$$
M A D_{\text {cur }}=\alpha_{1} * M A D_{\text {prev }}+\alpha_{2}
$$

where $M A D_{\text {cur }}$ and $M A D_{\text {prev }}$ represent respectively the current and the previous MAD of a given coding unit. $\alpha_{1}$ and $\alpha_{2}$ are the linear model coefficients. Once the predicted complexity $M A D_{\text {cur }}$ and a target bit-rate for a given P-frame (or Macroblock) (TBitsP) are determined, they are then included into a quadratic model used to compute the quantization parameter step $Q_{s t e p}$ :

$$
T \text { BitsP }=M A D_{\text {cur }} \times\left(\frac{x_{1}}{Q_{\text {step }}}+\frac{x_{2}}{Q_{\text {step }}^{2}}\right)
$$

where $x_{1}$ and $x_{2}$ are the first and second order coefficients. The Inter-rate control in H.264/AVC has also been the subject of several investigations. Efforts have been focused on the improvement of the MAD and its prediction through a linear model. The use of MAD, as complexity measure, handles image characteristics locally but in some cases remains inefficient, resulting in improper quantization parameter (QP) values. QP miscalculations may also result from the linear prediction model which assumes similar complexity from frame to frame. Like for the Intra-rate control, the proposed solutions are either applicable in a given context ${ }^{3} 1516$ or require more additional computations ${ }^{11}$.

In this paper, we propose a new R-Q model resulting from extensive experiments. This latter is divided into two models: An Intra R-Q model and an Inter one. The Intra R-Q model solves the initial QP problem by determining an optimal initial QP for Intra-frame while the Inter one overcomes problems related to MAD estimation and linear model. This latter substitutes both linear and quadratic models (Eq. 2 and Eq. 3) used in H.264/AVC rate control algorithm by a new logarithmic R-Q model. The global R-Q model (Inter and Intra models combined) does not need any complexity measure estimation. Objective and subjective simulations have been carried out using JM15.0 reference software $^{7}$. Compared to this latter, the global R-Q model improves the coding efficiency in terms of quality measured objectively (PSNR up to $+2.01 \mathrm{~dB}$ ), subjectively (by psycho visual experiments) and in terms of computational complexity. The rest of this paper is organized as follows. Section 2 describes in detail the proposed R-Q model. The experimental results are given in Section 3. Finally, we draw a conclusion in Section 4.

\section{PROPOSED R-Q MODEL}

As it was mentioned previously, our proposed R-Q model is divided into two models Intra and Inter R-Q models: 


\subsection{Intra R-Q model}

The improvement of the Intra-frame quality plays a key role in the enhancement of coding efficiency for all subsequent Inter-frames, because these latter are motion compensated directly or indirectly from the Intraframe. In H.264/AVC Intra-rate control, it is well known that the initial QP determination using the bits per pixel number (bpp) (Eq. 1) presents, at least, two defects: the range between QPs is large and the QPs values do not consider any frame feature (As the Flowchart in Figure 2 substantiates). To overcome these defects, a new R-Q model for Intra-frame is proposed. The first step of this algorithm, consists in determining a relationship between the target bit-rate of I-frame (TBitsI) and the fixed global target bit for the whole sequence (GlobalBitrate). To establish this relationship, three test sequences have been encoded (Bridge, Carphone and Foreman) at several low bit-rates ranging from $10 \mathrm{Kbps}$ to $80 \mathrm{Kbps}$. The actual bit-rates and two complexity measures (average gradient per pixel and Euclidean distance) of I-frame are computed. Then, the ratio between actual bit-rate and complexity measure of I-frame is plotted vs. global target bit. Whatever the complexity measure, the model which best fit the experimental data is the non linear model given by:

$$
\frac{\text { TBitsI }}{\delta}=a *\left(\frac{\text { GlobalBitrate }}{F r}\right)^{b}
$$

$\delta$ represents an I-frame complexity, $\mathrm{Fr}$ is the frame rate which equals to 30fps. The model parameters $a$ and $b$ are determined by the least squares method. Eight other sequences have served to confirm the accuracy of the above relationship (Akiyo, Claire, Grandma, Miss-America, Mother-Daughter, News, Salesman and Silent).

Once the ratio $R I / \delta$ is determined by the first model, we use a second model R-Q allowing the determination of the quantization parameter $(\mathrm{QP})$ of the Intra-frame without having to calculate its complexity $\delta$. To generate a relationship between the ratio $R I / \delta$ and $\mathrm{QP}$, we have used the same test conditions described above with an initial QP varying from 10 to 40 with a constant step of 2 . Fitting the experimental data $R I / \delta$ vs. QP leads to the non linear model of Eq. 5. This model allows an optimal determination of Intra-frame initial QP without having to calculate its complexity unlike methods described in $^{1} 1214$ :

$$
\frac{T \text { BitsI }}{\delta}=\frac{1}{\left(p_{1} * Q P^{2}+p_{2} * Q P+p_{3}\right)}
$$

where $p_{1}, p_{2}$ and $p_{3}$ are the Intra R-Q model coefficients.

\subsection{Inter R-Q Model}

The goal of a model-based QP is to develop a Rate-Quantization solution that intends to achieve more consistent video quality across the entire sequence for a large variety of sequences with different characteristics. In this section, we built a new Rate-Quantization model for Inter coding unit by analyzing the actual data generated by coding, using constant QPs across frames of the whole sequence. To achieve this goal, we performed a lot of experiments on various video sequences with various contents (Bridge, Carphone, Coastguard, Foreman, Highway and Suzie). These sequences are used to generate the new Inter R-Q model, and eight others (Akiyo, Claire, Grandma, Miss-America, Mother-Daughter, News, Salesman and Silent) are used to confirm its accuracy.

Each frame of the above test sequences is coded with constant QP ranging from 10 to 40 with a constant step of 2. The first frame is encoded as I and subsequent frames as P. In Figure 3, we plot the curves QPs versus bits per P-frames for three test sequences "Bridge", "Carphone" and "Foreman".

It can be seen from Figure 3 that there is a logarithmic relationship between QP and a target bit-rate (TBitsP) used to encode each P-frames in the sequence. This can be expressed as follows:

$$
Q P=\alpha * \log (\text { TargetBitsP })+\beta
$$

where $\alpha$ and $\beta$ are the model parameters which are defined by using a linear regression method that uses the coding statistics of the previously coded frames. As it can be shown, the proposed Inter R-Q model does not use any complexity measure unlike the H.264/AVC rate control algorithm which uses a linear model (Eq. 2) to predict the complexity measure and a quadratic model (Eq. 3) to compute the QP function of this complexity. 


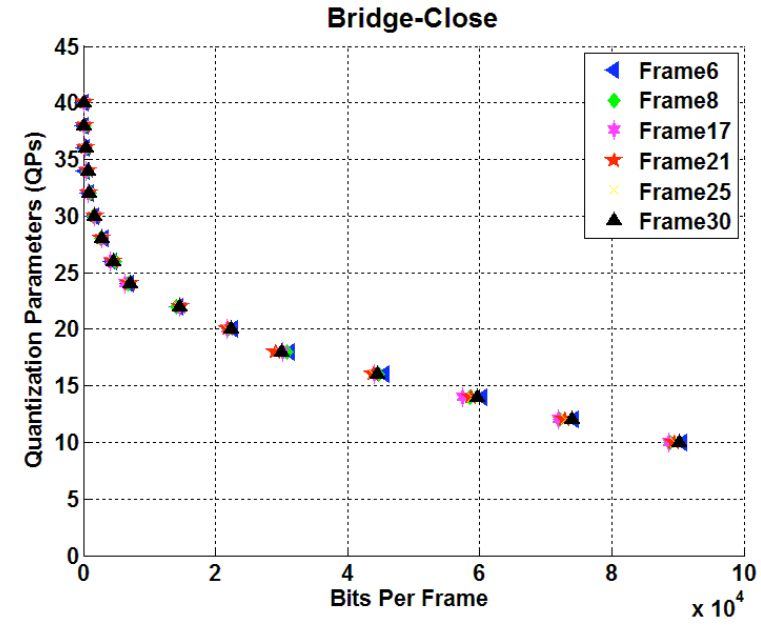

-a-

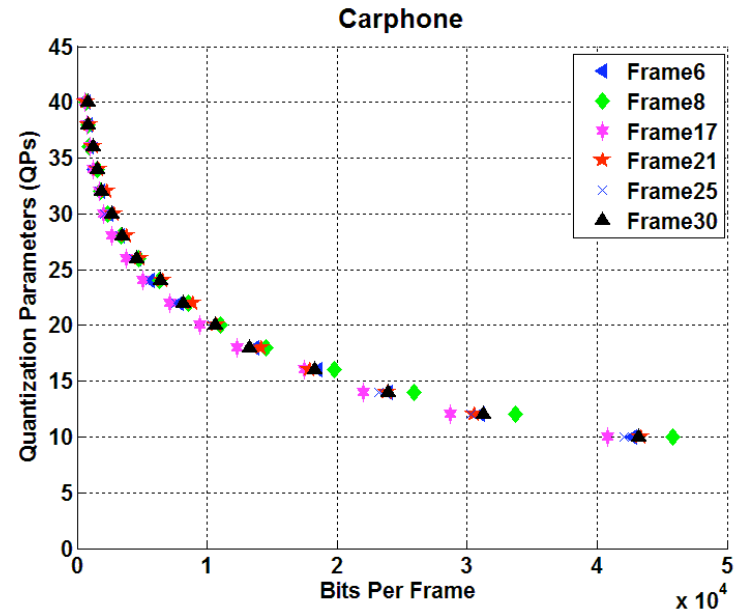

-b-

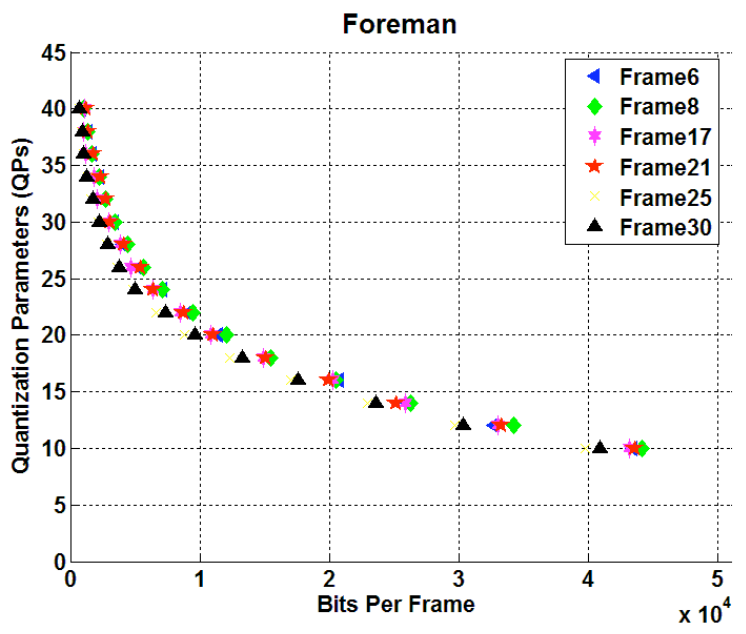

-c-

Figure 3. Rate-Quantization curves of some P-frames of (a):"Bridge", (b):"Carphone" and (c):"Foreman" sequences. 


\section{EXPERIMENTAL RESULTS}

The quality assessment has been performed at the macroblock level and the JVT reference software JM10.2 baseline profile ${ }^{7}$ has served for implementation. The proposed model R-Q has been implemented using the same software, so that all parts of the encoder except for rate control are the same. The computing system used for the experiments is composed by 2.16 GHZ Pentium 4 CPU and 2.00 GB RAM running Windows Vista. Fifteen common test sequences have been used (Akiyo, Bridge, Claire, Carphone, Container, Grandma, Highway, News...) in QCIF format (4:2:0) and 30 frames per second. Several global target bit-rates (RT) have been considered ranging from 15 to $64 \mathrm{Kbps}$. We have encoded the first frame as $\boldsymbol{I}$ and the subsequent frames as $\boldsymbol{P}$ (1 GOP of 90 frames). The JM15.0 $0^{7}$ reference software is used for comparison. This latter is one of the most recent versions of H.264/AVC encoder. It is used with the rate control mode set to "2" (rate control is applied with an intelligent QP selection for I and B slices). We evaluate the experimental results by both subjective and objective assessment.

\subsection{Subjective quality evaluation}

For the subjective quality evaluation, we used the Double-Stimulus Continuous Quality-Scale (DSCQS) methodology described in ITU recommendations ${ }^{5}$. The experiments were run in a dedicated room with neutral gray walls, a controlled D65 lighting, a calibrated display...15 observers with normal acuity and color vision have made the test. The scoring is performed on discrete scale composed of 5 quality levels: excellent, good, fair, poor and bad that correspond respectively to scores ranging from 100 to 0 . For this experiment, we preferred the usage of these values rather than 5 to 1 to simplify their interpretation.

Since the proposed Intra R-Q model determines an optimal initial QP for both Intra and the first P-frames, we subjectively evaluate their quality. Table 1 lists the evaluation results obtained by using DSCQS method when the Intra R-Q model is applied in comparaison with JM15.0. From Table 1, we can notice that the proposed R-Q model is visually efficient because it providers higher DSCQS scores in comparison to the JM15.0 and it implies at least a score gain of 12.8. It can be said that larger the score is, better is the obtained perceptual quality.

\subsection{Objective quality assessment}

In order to validate the effectiveness of the proposed R-Q model, we test this latter on image sequences, different from those used to generate it. We use average PSNR difference and average bit-rate difference as performance measures. $^{2}$ These measures are often used to compare R-D performance between two different methods. We also compare the initial QP values and the computational time with the JM15.0 reference software. The results are shown in Table 2.

Three remarks can be made from Table 2. Firstly, the initial QP values determined by the proposed R-Q model are smaller than those calculated by JM15.0 (In this case, only one value of QP is selected whatever the considered target bit-rates). Secondly, the proposed approach outperforms JM15.0 by improving the coding efficiency in terms of PSNR (up to $+2.01 \mathrm{~dB}$ ). Finally, the computational time is reduced (up to $+18 \mathrm{~s}$ ) because the proposed R-Q model incorporates an Inter R-Q one that substitutes both the linear and quadratic models used in JM15.0 and alleviates the memory occupation.

Figure 4 and Figure 5 give the Rate-Distortion and computational time versus target bit-rates for "Akiyo" and "Container" sequences. One can see that the proposed R-Q model achieves a higher PSNR and lower computational time in comparison with the JM15.0 rate control, for all selected bit-rates ranging from $15 \mathrm{Kbps}$ to $64 \mathrm{Kbps}$. The improvement of the R-D performance when our proposed approach is applied, enhances obviously the visual quality.

Finally, Figure 6 and Figure 7 depict the variation of the PSNR and the number of bits per P-frame for "Grandma" and "Salesman" sequences coded respectively at 20Kbps and 30Kbps. Figure 6 shows that for the proposed R-Q model, the PSNR variation in latter frames is lower than in earlier ones. However, the PSNR improvement of earlier frames is enough significant and leads to a more consistent quality with a lower variation of average PSNR/sequence (Average PSNR gain of $+1.5 \mathrm{~dB}$ in "Grandma" case and $+1.24 \mathrm{~dB}$ in "Salesman" 


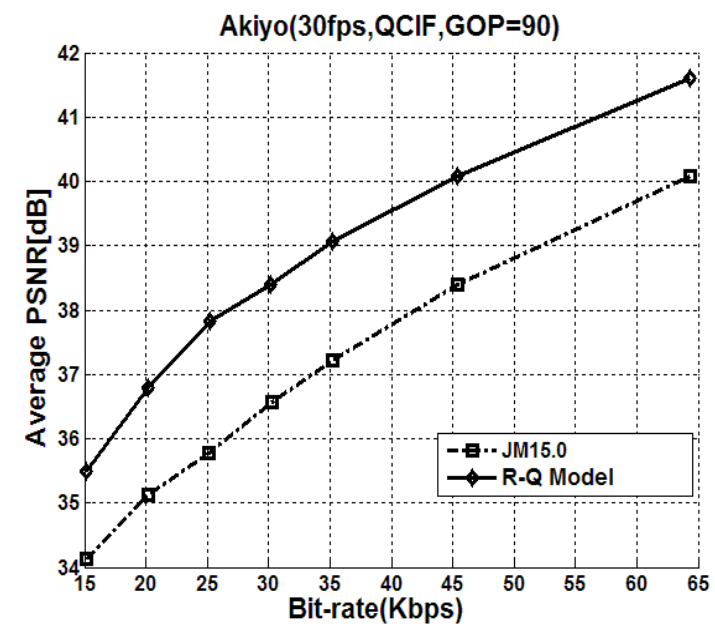

-a-

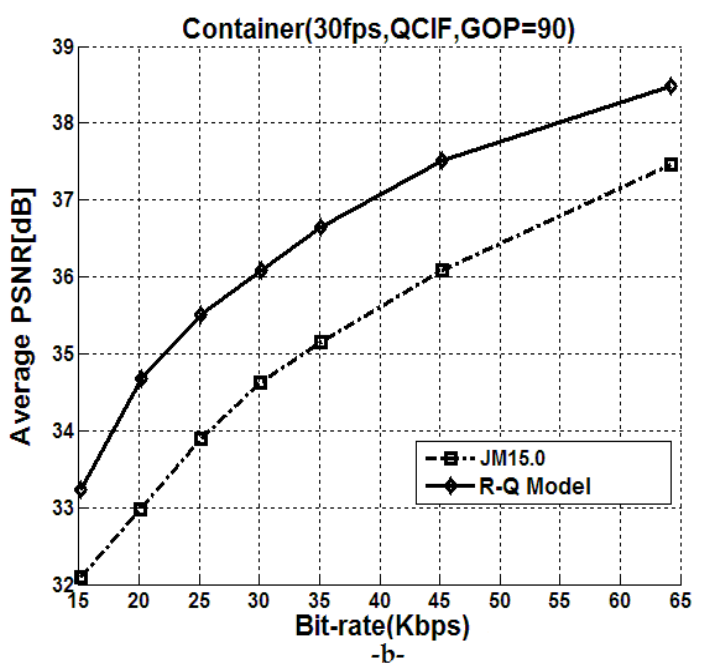

-b-

Figure 4. Rate-Distortion results for two sequences encoded at various bit-rates when our R-Q Model is applied and compared to the JM15.0 reference software for -a-:'Akiyo' and -b-:'Container'.
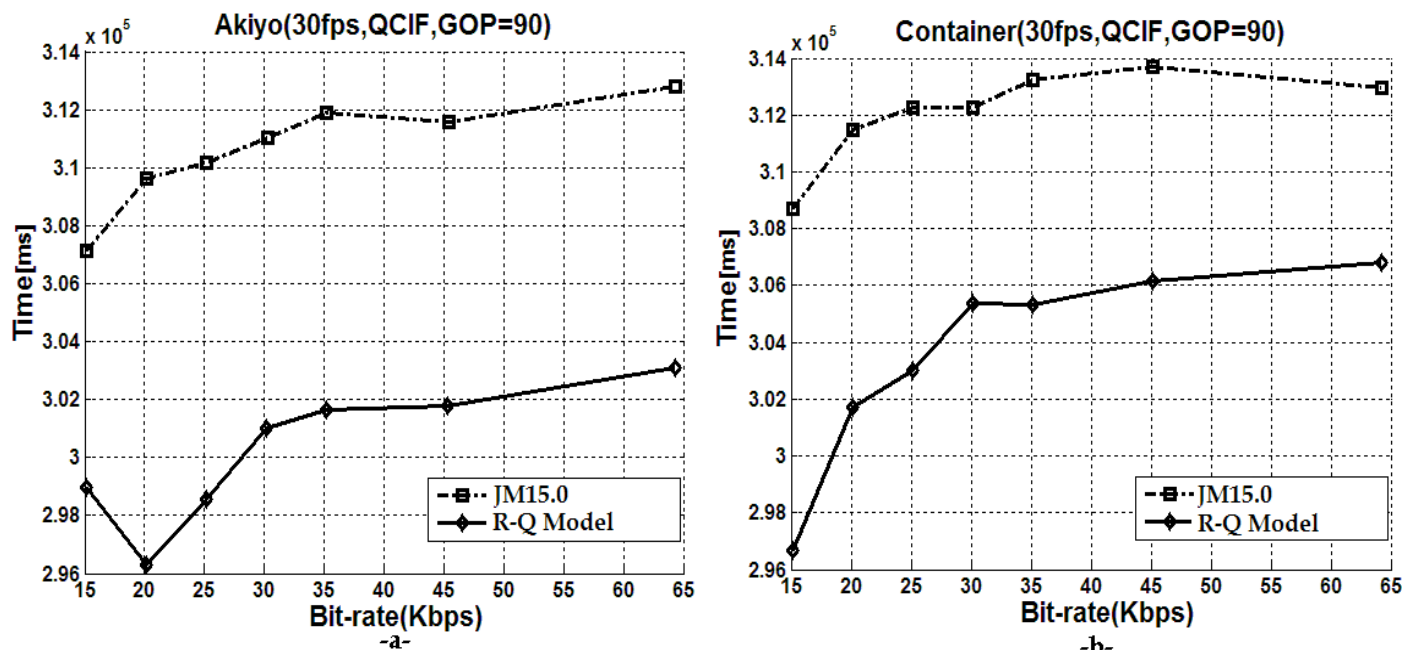

Figure 5. Time results for two sequences encoded at various bit-rates when our R-Q Model is applied and compared to the JM15.0 reference software for -a-:'Akiyo' and -b-:'Container'. 
Table 1. Subjective scores using DSCQS method for both Intra and the first P-frames when R-Q is applied in comparison with JM15.0.

\begin{tabular}{|c|c|c|c|c|c|}
\hline Sequences & $\begin{array}{c}\text { Target } \\
\text { Bit } \\
\text { Rate } \\
\text { (Kbps) }\end{array}$ & Frames & $\begin{array}{c}\text { DSCQS } \\
\text { Score } \\
\text { JM15.0 }\end{array}$ & $\begin{array}{c}\text { DSCQS } \\
\text { Score } \\
\text { R-Q } \\
\text { Model }\end{array}$ & $\begin{array}{l}\text { Score } \\
\text { Gain }\end{array}$ \\
\hline \multirow{7}{*}{ Akiyo } & 15 & I-Frame & 41 & 61,6 & 20,6 \\
\hline & \multirow[t]{2}{*}{20} & I-Frame & 40,2 & 65,7 & 25,5 \\
\hline & & P-Frame & 40,2 & 64,6 & 24,4 \\
\hline & \multirow[t]{2}{*}{30} & I-Frame & 41,3 & 66,4 & 25,1 \\
\hline & & P-Frame & 35,5 & 63,6 & 28,1 \\
\hline & \multirow[t]{2}{*}{45} & I-Frame & 40,1 & 71,8 & 31,7 \\
\hline & & P-Frame & 41,8 & 70,4 & 28,6 \\
\hline \multirow{8}{*}{ Container } & \multirow[t]{2}{*}{15} & I-Frame & 31,6 & 49 & 17,4 \\
\hline & & P-Frame & 36,2 & 49 & 12,8 \\
\hline & \multirow[t]{2}{*}{20} & I-Frame & 31,6 & 50,7 & 19,1 \\
\hline & & P-Frame & 36,7 & 59,4 & 22,7 \\
\hline & \multirow[t]{2}{*}{30} & I-Frame & 31,2 & 54,8 & 23,6 \\
\hline & & P-Frame & 31 & 56,1 & 25,1 \\
\hline & \multirow[t]{2}{*}{45} & I-Frame & 27,9 & 54 & 26,1 \\
\hline & & P-Frame & 33,7 & 52,3 & 18,6 \\
\hline \multirow{8}{*}{ News } & \multirow[t]{2}{*}{15} & I-Frame & 33,9 & 54,2 & 20,3 \\
\hline & & P-Frame & 31,5 & 51,1 & 19,6 \\
\hline & \multirow[t]{2}{*}{20} & I-Frame & 29,1 & 58,3 & 29,2 \\
\hline & & P-Frame & 30,7 & 53 & 22,3 \\
\hline & \multirow[t]{2}{*}{30} & I-Frame & 31,6 & 55,2 & 23,6 \\
\hline & & P-Frame & 25,2 & 55,3 & 30,1 \\
\hline & \multirow[t]{2}{*}{45} & I-Frame & $\overline{32,9}$ & 57,8 & 24,9 \\
\hline & & P-Frame & 33,8 & 60,8 & 27 \\
\hline \multirow{8}{*}{ Salesman } & \multirow[t]{2}{*}{15} & I-Frame & 29,8 & 47,9 & 18,1 \\
\hline & & P-Frame & 28,9 & 50,5 & 21,6 \\
\hline & \multirow[t]{2}{*}{20} & I-Frame & 29,8 & 62,7 & 32,9 \\
\hline & & P-Frame & 35,6 & 57,7 & 22,1 \\
\hline & \multirow[t]{2}{*}{30} & I-Frame & 40,6 & 64,6 & 24 \\
\hline & & P-Frame & 34,7 & 61,2 & 26,5 \\
\hline & \multirow[t]{2}{*}{45} & I-Frame & 34,5 & 57,8 & 23,3 \\
\hline & & P-Frame & 27,7 & 56,2 & 28,5 \\
\hline
\end{tabular}

case).

Figure 7 shows the actual bits ditribution among P-Frames for the same sequences. It can be seen that the bits/frame variation is lower for the proposed approach. Moreover, the average bits/frame of our algorithm remains lower than the average target bits/frame.

\section{CONCLUSION}

In this paper, we have proposed a new Rate-Quantization model. This latter aims at overcoming some defects related to H.264/AVC rate controller for both Inter and Intra-frames. Our proposed approach resolves firstly, the issue of inappropriate calculation of the initial QP for I-frame by determining efficiently, an optimal initial QP according to both target bit-rate and complexity of the I-frame without having to determine this latter. Secondly, it overcomes the problems related to the use of MAD measure and the linear model by proposing a new logarithmic R-Q model for Inter coding unit (Frame or Macroblock). The logarithmic model substitutes both the linear and quadratic models used in H.264/AVC rate controller, and does not need any complexity measure. Objective and subjective experiments show that the proposed approach enhances significantly the R-D performance in lower bit-rate by reducing computational time and improving the sequence quality (in term of consistence and average PSNR). In order to include further improvement in the visual quality, we aim to improve 

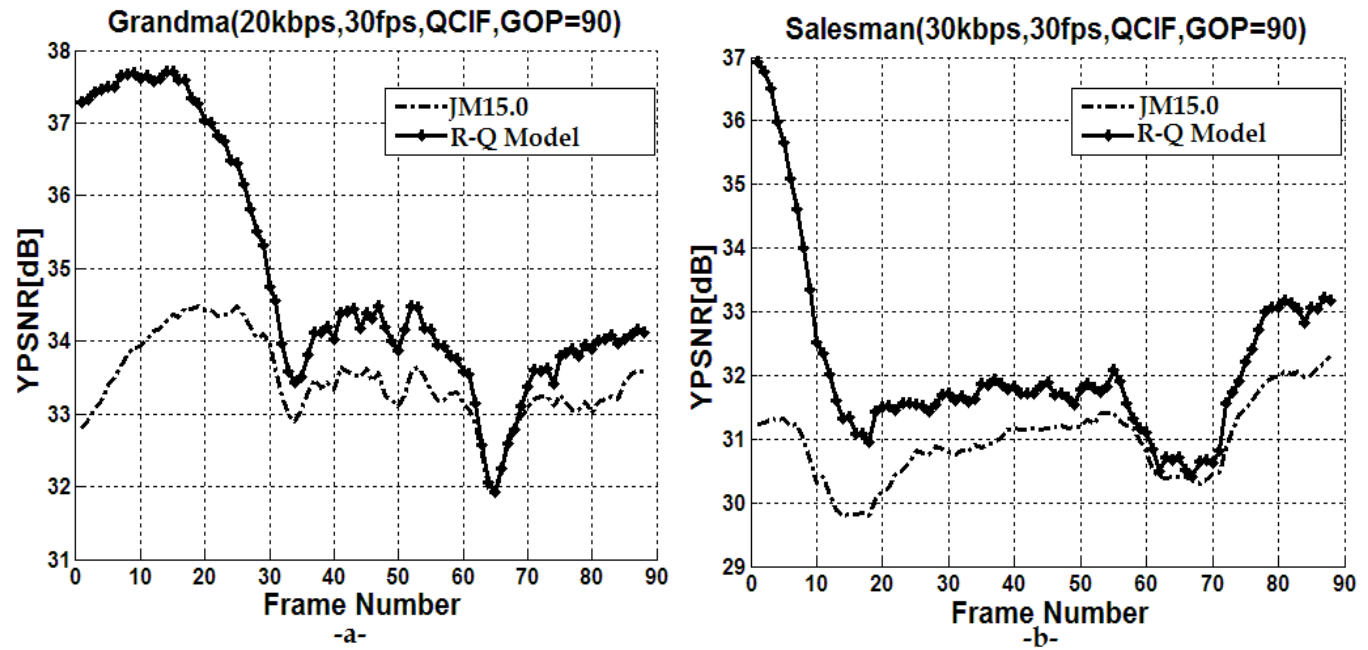

Figure 6. PSNR variations per P-Frames for 2 sequences when our R-Q Model is applied and compared with the JM15.0. 'Grandma' encoded at 20Kbps':(-a-) and 'Salesman' encoded at 30Kbps':(- $\boldsymbol{b}-\mathbf{-})$.
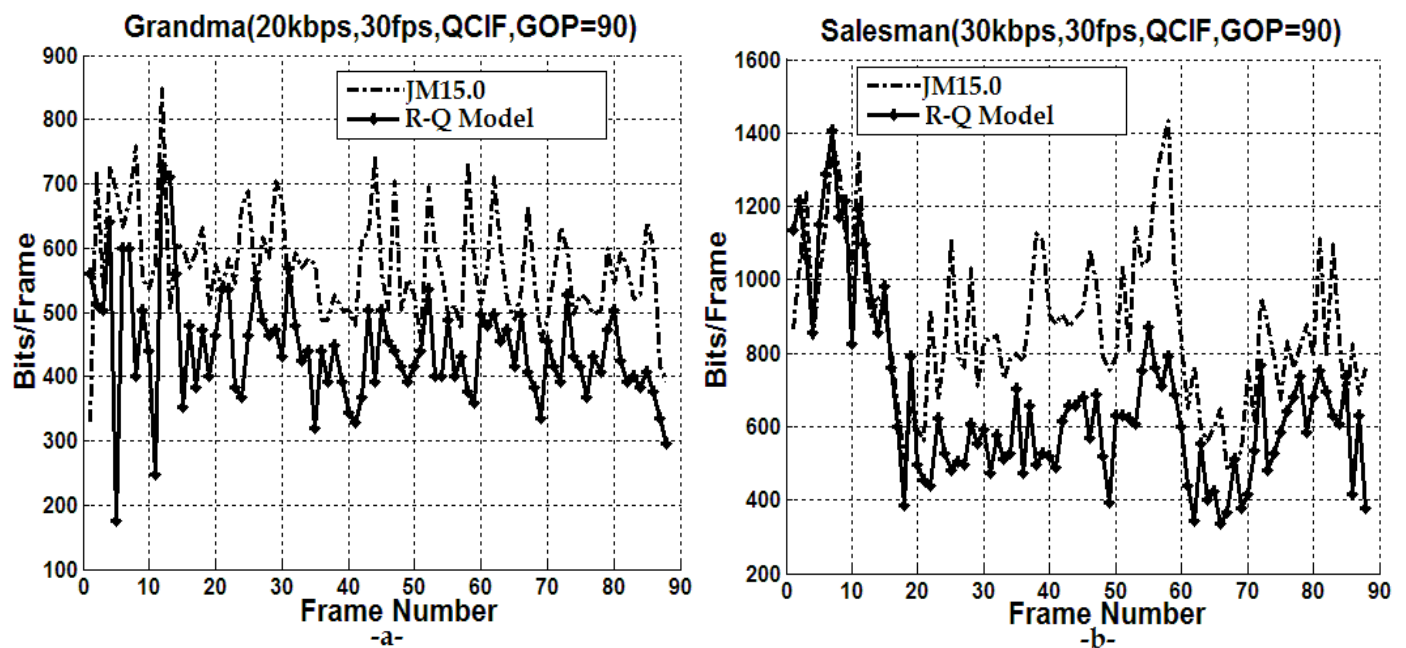

Figure 7. Bit-rate variations per P-Frames for 2 sequences when our R-Q Model is applied and compared with the JM15.0. 'Grandma' encoded at 20Kbps':(-a-) and 'Salesman' encoded at 30Kbps':(- $\boldsymbol{b}-)$. 
the bit allocation process at frame and macroblock layers by including some complexity measures able to allocate bits according to frame/macroblock characteristics.

\section{REFERENCES}

[1] F. Pan et al., "An intra-frame quantization optimization algorithm for very low bit-rate video coding". Inst. for Infocomm Research, Tech. Rep, June 2002, Singapore.

[2] G. Bjontegaard, "Calculation of Average PSNR Differences between RD-Curves", ITU-T Q.6/SG16 VCEG 13th Meeting, Document VCEG-M33, Austin, USA, Apr. 2001.

[3] I-H. Shina, Y-L. Leeb, H-W. Parka," Rate control using linear rate- $\rho$ model for H.264/AVC". Signal Processing: Image Com., vol. 19, pp. 341-352, April 2004.

[4] ISO/IEC JTCI/SC29/WG11, Test Model 5, 1993.

[5] ITU-R, Methodology for the Subjective Assessment of the Quality of Television Pictures, ITU-R Recommendation BT.500-11, Mar. 2000.

[6] ITU-T/SG15, Video Codec Test Model, TMN8, Portland, June 1997.

[7] JM10.2,JM15.0, http://iphome.hhi.de/suehring/tml /download/ jm10.2.zip,jm15.0.zip.

[8] J. Ribas-Corbera, S. Lei, "Rate control in DCT video coding for low-delay communications". IEEE Trans. Circuits Syst. Video Technol., vol. 9, no. 1, pp 172-185,Feb 1999.

[9] K. P. Lim, G. Sullivan, T. Wiegand, "Text description of joint model reference encoding methods and decoding concealment methods". Joint Video Team of ISO/IEC JTC1/SC29/WG11 And ITU-T SG16/Q.6 Doc. JVT-N046,Hong Kong, China, January 2005.

[10] MPEG-4 Video Verification Model V18.0, Coding of Moving Pictures and Audio N3908, ISO/IEC, JTC1/SC29/ WG11, Jan. 2001.

[11] N. Kamaci, Y. Altunbasak, R. M. Mersereau,"Frame bit allocation for the H.264/AVC video coder via Cauchy-Density-Based rate and distortion models". IEEE Trans. Circuits Syst. Video Technol., vol. 15, no. 8, pp 994-1006, Aug 2005.

[12] S. Zhou et al, "Improvement on rate-distortion performance of H.264/AVC rate control in low bit rate". IEEE Trans. Circuits Sys. Video Technol, Vol. 17, N 8, pp. 996-1006, Aug. 2007.

[13] T. Wiegand, G.J. Sullivan, G. Bjontegaard, and A. Luthra, "Overview of the H.264/AVC video coding standard". IEEE Trans. Circuits Systems Video Technol, pp. 560576, 13 (2003).

[14] X. Jing and L-P. Chau," A Novel Intra-Rate Estimation Method for H.264/AVC Rate Control". IEEE International Symposium on Circuits and Systems (ISCAS), 2006.

[15] X. Yi and N. Ling, "Improved H.264/AVC rate control by enhanced MAD-based frame complexity prediction". Journal of Visual Communication and Image Representation, Volume 17, Issue 2, April 2006, Pages 407-424.

[16] Y. Ming, X. Yun, G. Fen, C. Shuting, "Improvements on MB-layer Rate Control Scheme for H.264/AVC video Using complexity estimation". Eighth ACIS International Conference on Software Engineering, Artificial Intelligence, Networking, and Parallel/Distributed Computing (SNPD 2007),vol. 2, pp.280284,2007

[17] Z. G. Li, F. Pan, K. P. Lim, G. Feng, X. Lin, and S. Rahardja," Adaptive Basic Unit Layer Rate Control for JVT". ISO/IEC JTC1/SC29/WG11 and ITU-T SG16 Q.6-G012, Thailand 2003.

[18] Z. He, Y. K. Kim and S. K. Mitra, "Low delay rate control for DCT video coding via p domain source modeling". IEEE Trans. Circuits Syst. Video Technol, vol. 11, no 8, pp 928-940, Aug 2001. 
Table 2. Comparison of initial QP values, Average PSNR, Average Bit-Rates and Computational Time Differences between the R-Q Model and JM15.0 reference software.

\begin{tabular}{|c|c|c|c|c|c|c|}
\hline Sequences & $\begin{array}{l}\text { Target } \\
\text { Bit-Rate } \\
\text { (Kbps) }\end{array}$ & $\begin{array}{l}\text { Methods } \\
\text { and } \\
\text { Gain }\end{array}$ & $\begin{array}{c}\text { Initial QP } \\
\text { Value }\end{array}$ & $\begin{array}{l}\text { Average } \\
\text { PSNR } \\
\text { (dB) }\end{array}$ & $\begin{array}{c}\text { Average } \\
\text { Bit-Rate } \\
\text { (Kbps) }\end{array}$ & $\begin{array}{c}\text { Computational } \\
\text { Time } \\
\text { (s) }\end{array}$ \\
\hline \multirow{4}{*}{ Akiyo } & 15 & $\begin{array}{c}\text { JM15.0 } \\
\text { R-Q Model } \\
\text { Gain }\end{array}$ & $\begin{array}{l}35 \\
29 \\
-\end{array}$ & $\begin{array}{r}34.14 \\
35.49 \\
+1.35\end{array}$ & $\begin{array}{c}15.14 \\
15.14 \\
0\end{array}$ & +8.17 \\
\hline & 20 & $\begin{array}{l}\text { JM15.0 } \\
\text { R-Q Model } \\
\text { Gain }\end{array}$ & $\begin{array}{l}35 \\
28 \\
-\end{array}$ & $\begin{array}{l}35.12 \\
36.79 \\
+1.67\end{array}$ & $\begin{array}{l}20.17 \\
20.18 \\
-0.01\end{array}$ & +13.337 \\
\hline & 30 & $\begin{array}{c}J M 15.0 \\
\text { R-Q Model } \\
\text { Gain }\end{array}$ & $\begin{array}{l}35 \\
27 \\
- \\
\end{array}$ & $\begin{array}{l}36.56 \\
38.40 \\
+1.84\end{array}$ & $\begin{array}{l}30.26 \\
30.22 \\
+0.04\end{array}$ & +10.011 \\
\hline & 64 & $\begin{array}{c}\text { JM15.0 } \\
\text { R-Q Model } \\
\text { Gain }\end{array}$ & $\begin{array}{l}35 \\
24 \\
-\end{array}$ & $\begin{array}{l}40.08 \\
41.60 \\
+1.52\end{array}$ & $\begin{array}{l}64.38 \\
64.31 \\
+0.07\end{array}$ & +9.705 \\
\hline \multirow{4}{*}{ Container } & 15 & $\begin{array}{c}\text { JM15.0 } \\
\text { R-Q Model } \\
\text { Gain }\end{array}$ & $\begin{array}{c}35 \\
29 \\
-\end{array}$ & $\begin{array}{l}32.10 \\
33.23 \\
+1.13\end{array}$ & $\begin{array}{l}15.13 \\
15.14 \\
-0.01\end{array}$ & +12.028 \\
\hline & 20 & $\begin{array}{c}\text { JM15.0 } \\
\text { R-Q Model } \\
\text { Gain }\end{array}$ & $\begin{array}{l}35 \\
28 \\
-\end{array}$ & $\begin{array}{l}32.97 \\
34.67 \\
+1.7\end{array}$ & $\begin{array}{l}20.13 \\
20.15 \\
-0.02\end{array}$ & +9.777 \\
\hline & 30 & $\begin{array}{c}\text { JM15.0 } \\
\text { R-Q Model } \\
\text { Gain }\end{array}$ & $\begin{array}{c}35 \\
27 \\
-\end{array}$ & $\begin{array}{l}34.62 \\
36.09 \\
+1.47\end{array}$ & $\begin{array}{l}30.12 \\
30.15 \\
-0.03\end{array}$ & +6.878 \\
\hline & 45 & $\begin{array}{c}\text { JM15.0 } \\
\text { R-Q Model } \\
\text { Gain }\end{array}$ & $\begin{array}{c}35 \\
25 \\
-\end{array}$ & $\begin{array}{l}36.09 \\
37.52 \\
+1.43\end{array}$ & $\begin{array}{c}45.19 \\
45.19 \\
0\end{array}$ & +7.546 \\
\hline \multirow{4}{*}{ News } & 15 & $\begin{array}{c}\text { JM15.0 } \\
\text { R-Q Model } \\
\text { Gain }\end{array}$ & $\begin{array}{l}35 \\
29 \\
-\end{array}$ & $\begin{array}{c}29.61 \\
29.61 \\
0\end{array}$ & $\begin{array}{l}15.89 \\
15.33 \\
+0.56\end{array}$ & +12.91 \\
\hline & 20 & $\begin{array}{c}\text { JM15.0 } \\
\text { R-Q Model } \\
\text { Gain }\end{array}$ & $\begin{array}{l}35 \\
28 \\
-\end{array}$ & $\begin{array}{l}30.46 \\
30.96 \\
+0.5\end{array}$ & $\begin{array}{l}20.26 \\
20.22 \\
+0.04\end{array}$ & +12.366 \\
\hline & 25 & $\begin{array}{c}\text { JM15.0 } \\
\text { R-Q Model } \\
\text { Gain }\end{array}$ & $\begin{array}{c}35 \\
27 \\
-\end{array}$ & $\begin{array}{l}31.20 \\
32.27 \\
+1.07\end{array}$ & $\begin{array}{l}25.23 \\
25.18 \\
+0.05\end{array}$ & +13.476 \\
\hline & 45 & $\begin{array}{c}\text { JM15.0 } \\
\text { R-Q Model } \\
\text { Gain }\end{array}$ & $\begin{array}{c}35 \\
25 \\
-\end{array}$ & $\begin{array}{l}33.27 \\
35.28 \\
+2.01\end{array}$ & $\begin{array}{l}45.35 \\
45.37 \\
+0.02\end{array}$ & +12.404 \\
\hline \multirow{4}{*}{ Salesman } & 20 & $\begin{array}{c}\text { JM15.0 } \\
\text { R-Q Model } \\
\text { Gain }\end{array}$ & $\begin{array}{c}35 \\
28 \\
-\end{array}$ & $\begin{array}{l}29.62 \\
30.16 \\
+0.54\end{array}$ & $\begin{array}{l}20.17 \\
20.15 \\
+0.02\end{array}$ & +17.447 \\
\hline & 25 & $\begin{array}{c}\text { JM15.0 } \\
\text { R-Q Model } \\
\text { Gain }\end{array}$ & $\begin{array}{l}35 \\
27 \\
-\end{array}$ & $\begin{array}{l}30.43 \\
31.35 \\
+0.92\end{array}$ & $\begin{array}{l}25.15 \\
25.16 \\
-0.01\end{array}$ & +18.002 \\
\hline & 30 & $\begin{array}{c}\text { JM15.0 } \\
\text { R-Q Model } \\
\text { Gain }\end{array}$ & $\begin{array}{c}35 \\
27 \\
-\end{array}$ & $\begin{array}{l}30.98 \\
32.22 \\
+1.24\end{array}$ & $\begin{array}{c}30.19 \\
30.19 \\
0\end{array}$ & +17.064 \\
\hline & 35 & $\begin{array}{l}J M 15.0 \\
\text { R-Q Model } \\
\text { Gain }\end{array}$ & $\begin{array}{r}35 \\
26 \\
- \\
\end{array}$ & $\begin{array}{r}31.63 \\
33.04 \\
+1.41 \\
\end{array}$ & $\begin{array}{l}35.20 \\
35.18 \\
+0.02 \\
\end{array}$ & +16.498 \\
\hline
\end{tabular}

